\title{
On Enhancing the Pilot-Aided Sampling Clock Offset Estimation of Mobile OFDM Systems
}

\author{
Cássio F. Dantas, Davi A. L. Castro, Cristiano M. Panazio
}

\begin{abstract}
Sampling Clock Offset (SCO) estimation is an important issue in Orthogonal Frequency-Division Multiplexing (OFDM) systems because sampling frequency mismatch between the transmitter and the receiver may severely degrade the system performance due to the loss of orthogonality between the subcarriers. SCO estimation in mobile environment is quite challenging since channel variation leads to an additional phase rotation that masks the SCO effects. However, most of the existing techniques rely on the assumption of a time-invariant channel and become considerably inaccurate in a mobile environment. In this paper, we propose an improvement to an existing pilot-aided SCO estimator aiming to provide robustness against channel variations. Performance was evaluated through simulations in an ISDB-T (Integrated Services Digital Broadcasting Terrestrial) compliant system and the results have shown a considerable improvement for all ranges of signal-to-noise ratios, specially for higher Doppler spread.
\end{abstract}

Index Terms-OFDM, Sampling Clock Offset, Sampling Frequency Offset, Time-varying Channels, Doppler spread, ISDB-T system.

\section{INTRODUCTION}

Orthogonal Frequency-Division Multiplexing (OFDM) is a multi-carrier technique that achieves high spectrum efficiency by dividing data into closely spaced subcarriers which constitute parallel orthogonal data-streams. It has been adopted in several broadband communication systems such as digital TV standards DVB [2] and ISDB [3].

It is known that the performance of OFDM systems is very sensitive to a precise synchronization. Sampling Clock Offset (SCO), also called Sampling Frequency Offset (SFO), occurs when the receiver's sampling frequency does not correspond to the true value. It is a central issue in practical implementations since such impairment leads to the loss of orthogonality between the subcarriers, i.e. Inter-Carrier Interference (ICI), causing performance degradation. The effects are intensified when larger OFDM symbols are used, as it is the case on digital TV standards.

A variety of SCO estimators have been proposed so far [4]-[10], the post-fast Fourier transform (FFT) methods being more widespread than the pre-FFT ones. Most of the post-FFT estimators use the pilots of adjacent symbols to identify the phase rotation caused by the SCO [4]-[7]. In those

The Associate Editors coordinating the review of this manuscript and approving it for publication were Profs. Cecílio Pimentel and Marcelo da Silva Pinho.

Cássio F. Dantas and Davi A. L. Castro are with Idea! Electronic Systems. Cristiano M. Panazio is with University of São Paulo. E-mails: \{cassio.fraga,davi.castro\}@idea-ip.com, cpanazio@usp.br

A preliminary version of this paper was presented in XXXIII Simpósio Brasileiro de Telecomunicações (SBrT'15), Juiz de Fora, MG, Brazil September 1-4, 2015 [1].

Digital Object Identifier: 10.14209/jcis.2016.10 methods, the channel is assumed to be time-invariant or at least slow time-variant between a few OFDM symbols, so that the phase distortions introduced in each subcarrier can be canceled without interfering with the SCO estimation. A different pilot-based post-FFT technique proposed in [8] tries to detect the shift on the estimated channel impulse response (CIR) caused by SCO. Although it is designed to work in time-varying channels, it might result in a low resolution estimation depending on the system parameters. Pre-FFT methods have been proposed in [9], [10]. They perform a non data aided estimation by relying on the statistical particularities of the time-domain OFDM signal, typically using the cyclic prefix. However, the performance of the method in [9] is degraded by multipath channels, and both of them, similar to the previous ones, cannot cope with time-varying channels.

Based on a conventional pilot-aided SCO estimator, we propose two new algorithms that mitigates the perturbations of frequency-selective time-varying channels. Note that the underlying algorithm is still affected by the channel variations, but we establish heuristics to identify the most distorted data and prevent them from degrading the estimation.

It is worth noting that these two new methods can provide additional robustness over our previously proposed technique described and analyzed in [1]. We also provide in this paper a deeper and a more detailed analysis of the proposed methods, including a convergence rate assessment through simulations as well as bit error rate (BER) results, which were not evaluated in [1]. The proposed estimators show, for frequency selective time-varying channels, faster convergence with none or little additional complexity.

This paper is organized as follows. In section II the OFDM system model is described, as well as the effects of the SCO on the received signal. Section III is divided in two parts: first, we briefly present the conventional pilot-aided SCO estimator in which our proposal is based; then, we describe the proposed estimators that aim for stable and robust operation on mobile environment. The simulation results and analysis are presented in section IV and conclusions in section V.

\section{System Model}

Consider an OFDM system in which the transmitted baseband signal is given by [11]:

$$
s(t)=\frac{1}{\sqrt{T_{u}}} \sum_{l=-\infty}^{+\infty} \sum_{k=-K / 2}^{+K / 2} a_{l, k} \psi_{l, k}(t),
$$

where $a_{l, k}$ denotes the data (or pilot) symbols, with $k$ being the subcarrier frequency index and $l$ being the OFDM symbol time index, $K+1$ (with $K$ even) is the total number of data symbols 
on each OFDM symbol and $\psi_{l, k}(t)$ denotes the subcarrier pulses with baseband frequency $f_{k}=k / T_{u}$, where $T_{u}$ is the useful time period where information can be transmitted.

We also assume that the OFDM symbol has a guard interval of duration $T_{g}$ appended to its prefix to retain the orthogonality after passing through time dispersive channels, so that we can define the subcarriers pulses as:

$$
\begin{aligned}
\psi_{l, k}(t) & =\exp \left(j 2 \pi\left(\frac{k}{T_{u}}\right)\left(t-T_{g}-l T_{s}\right)\right) u\left(t-l T_{s}\right) \\
u(t) & = \begin{cases}1, & 0 \leq t<T_{s} \\
0, & \text { else }\end{cases}
\end{aligned}
$$

where $T_{s}=T_{u}+T_{g}$ is the resulting symbol period, which is equivalent to $N_{s}=N+N_{g}$ samples for a sampling period equal to $T=T_{s} / N_{s}$, where $N$ is IFFT size and $N_{g}$ is the cyclic prefix length.

The signal is then transmitted over a frequency selective fading channel which equivalent channel impulse response is time-varying and can be represented by:

$$
h(\tau, t)=\sum_{i} h_{i}(t) \delta\left(\tau-\tau_{i}\right),
$$

where $\tau_{i}$ is the delay of the $i$-th path and $h_{i}(t)$ is a complex Gaussian stochastic process with a variance $\sigma_{h_{i}}^{2}$ and correlation described by the Jakes' model [12].

The received signal is

$$
r(t)=\sum_{i} h_{i}(t) s\left(t-\tau_{i}\right)+\tilde{\eta}(t)
$$

where $\tilde{\eta}(t)$ is a white Gaussian noise with power spectral density $N_{0} / 2$, and then it is passed through an ideal low pass filter with cutoff frequency $\frac{N}{2 T s}$ and sampled at timing instants $t_{n}=n T^{\prime}$, where $T^{\prime}$ is the sampling period at the receiver, yielding

$$
r\left(t_{n}\right)=\sum_{i} h_{i}\left(n T^{\prime}\right) s\left(n T^{\prime}-\tau_{i}\right)+\eta\left(n T^{\prime}\right),
$$

where $\eta\left(n T^{\prime}\right)$ is the filtered noise assumed to be an additive complex white Gaussian noise with variance $\sigma_{\eta}^{2}$.

After removing the guard interval, the $n$-th sample for the $l$-th received symbol is represented by

$$
r_{l, n}=r\left(\left(n+N_{g}+l N_{s}\right) T^{\prime}\right)=r\left(n^{\prime} T^{\prime}\right),
$$

with the abbreviation $n^{\prime}=n+N_{g}+l N_{s}$.

In case of perfect synchronization $\left(T^{\prime}=T\right)$ and assuming the channel to be constant during the transmission of one OFDM symbol, the demodulation of the signal through the FFT gives

$$
z_{l, k}=a_{l, k} H_{l, k}+\eta_{l, k},
$$

where $H_{l, k}$ is the channel frequency response given by the FFT of the corresponding channel impulse response $\left\{h_{i}(l) \exp \left(-j 2 \pi \tau_{i} l / N\right)\right\}$ and $\eta_{l, k}$ is the FFT of $\left\{\eta\left(n^{\prime} T^{\prime}\right)\right\}$.

a) Fast-Fading Channel: If the channel varies within one OFDM symbol, the frequency domain received signal can be represented by

$$
z_{l, k}=a_{l, k} H_{l, k}+\eta_{l, k}+\eta_{I C I ; l, k} .
$$

where $\eta_{I C I ; l, k}$ is the interference generated by ICI, which is caused by the time-varying channel that breaks the subcarriers orthogonality. Such inteference can be modeled, if needed, as additive Gaussian noise [13]. Additionally, strictly speaking, $H_{l, k}$ no longer represents the physical channel at a certain time instant but rather the result of an averaging over a time period equal to $T_{s}$.

b) Sampling Clock Offset: In the case where $T^{\prime} \neq T$, we can define the relative $\mathrm{SCO}$ as

$$
\zeta=\left(T^{\prime}-T\right) / T \text {. }
$$

We suppose that no other synchronization error is present. By substituting (1) in (5), the time domain signal may be written as

$$
r_{l, n}=\sum_{i} h_{i}\left(n T^{\prime}\right) \sum_{l} \sum_{k} a_{l, k} \psi_{l, k}\left(n^{\prime} T^{\prime}-\tau_{i}\right)+\eta\left(n^{\prime} T^{\prime}\right) .
$$

After the demodulation via FFT, the frequency domain samples become [11]

$$
\begin{aligned}
z_{l, k}= & {\left[\exp \left(j \pi \phi_{k}\right) \cdot \exp \left(j 2 \pi \phi_{k}\left(l N_{s}+N_{g}\right) / N\right)\right] } \\
& \operatorname{sinc}\left(\pi \phi_{k}\right) a_{l, k} H_{l, k} \\
& +\sum_{\substack{l \neq k\\
}}\left[\exp \left(j \pi \phi_{i, k}\right) \cdot \exp \left(j 2 \pi \phi_{i}\left(l N_{s}+N_{g}\right) / N\right)\right] \\
& \operatorname{sinc}\left(\pi \phi_{i, k}\right) a_{l, i} H_{l, i}+\eta_{I C I ; l, k}+\eta_{l, k},
\end{aligned}
$$

where

$$
\begin{aligned}
\phi_{i, k} & =(1+\zeta) i-k, \\
\phi_{k} & =\phi_{k, k}=\zeta k .
\end{aligned}
$$

A first remarkable effect is the appearance of new additive ICI term, i.e., the second term of (11). This term is analogous to $\eta_{I C I}$ and is composed of the summation of the product of random variables and one of them is the zero-mean independent identically distributed $a_{l, k}$ symbols, so that they are uncorrelated and also have zero mean, thus, being usually modeled as a Gaussian variable [13]. Hence, for simplicity it will be incorporated into the noise term $\eta_{I C I}$ from the time-varying channel scenario.

Some further simplifications can be performed on (11). For practical deviations $\left(\phi_{k}<<1\right)$ the multiplicative term $\operatorname{sinc}\left(\pi \phi_{k}\right) \approx 1$ so that it can be neglected. Likewise, the time invariant term $\exp \left(j \pi \phi_{k}\right)$ can be incorporated into the channel gain factor $H_{l, k}$. We are left with

$z_{l, k}=a_{l, k} H_{l, k} \exp \left(\frac{j 2 \pi k \zeta}{N}\left(l N_{s}+N_{g}\right)\right)+n_{l, k}+n_{I C I ; l, k}$.

Therefore, the main effect of SCO on the received symbols - set aside ICI - is a phase rotation that linearly grows with the subcarrier index $k$. The slope of this linear phase distortion is directly proportional to the relative $\operatorname{SCO}(\zeta)$, which will be estimated by the algorithms presented in section III.

\section{SCO ESTIMATION}

Pilot symbols are used in the estimation process. In this paper we adopt a pilot structure compatible with ISDB-T 


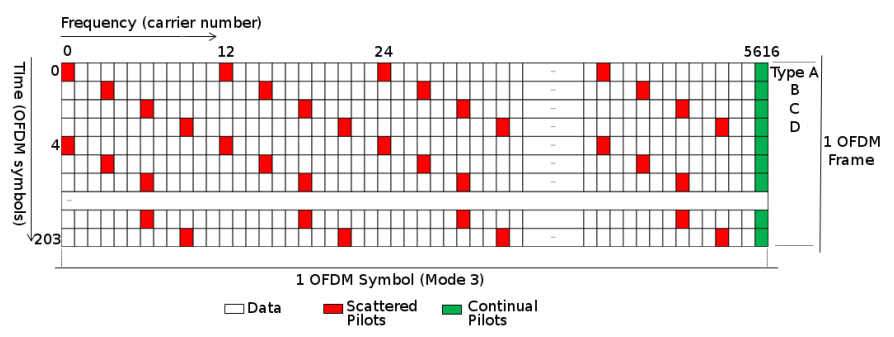

Fig. 1. Pilot pattern on ISDB-T systems.

systems, which is represented in Fig. 1. Each OFDM symbol may contain one of the four different types of scattered pilots - A,B,C and D in sequence - characterized by the subcarrier index of the first pilot. Within one symbol the pilots are equally spaced by 12 subcarriers. The pilot content is a BPSK (Binary Phase-Shift Keying) symbol following a PRBS (Pseudo Random Binary Sequence) defined in [3]. Nevertheless, the following algorithms presented in this paper are not restricted to this specific pilot pattern, being applicable to other pilot spacings both in time and frequency domains.

\section{A. Conventional Method}

Considering equation (13) and neglecting the noise terms, it remains impossible to estimate the phase rotation on the pilot symbols because of the unknown channel gain term $H_{l, k}$. As a matter of fact, in most practical systems the synchronization block precedes the channel estimation.

The main idea of the method proposed in [5] is to eliminate the effects of the channel gain term by taking the phase difference between two pilots in different symbols that hold the same subcarrier index. This can be achieved by taking the product with the complex conjugate of the second pilot.

$$
\begin{aligned}
& Y_{D, k}=z_{l+D, k} \cdot z_{l, k}^{*} \\
& =\left[a_{l+D, k} H_{l+D, k} \exp \left(j 2 \pi k \zeta\left((l+D) N_{s}+N_{g}\right) / N\right)\right] \\
& \cdot\left[a_{l, k}^{*} H_{l, k}^{*} \exp \left(-j 2 \pi k \zeta\left(l N_{s}+N_{g}\right) / N\right)\right] \\
& =\left|a_{l, k}\right|^{2}\left|H_{l, k}\right|^{2} \exp \left(j 2 \pi k \zeta D N_{s} / N\right),
\end{aligned}
$$

where $D$ is the distance in OFDM symbols between the pilots of the same type (e.g., see Fig. 1).

In the ISDB-T standard, the pilot structure is so that $D=4$, in which $a_{l, k}=a_{l+D, k}$. Also note that in (14) the channel is assumed static between the two symbols where the pilots are located, i.e. $H_{l, k}=H_{l+D, k}$ so that the product $H_{l+D, k} H_{l, k}^{*}=H_{l, k} H_{l, k}^{*}=\left|H_{l, k}\right|^{2}$. Furthermore, the phase of $Y_{D, k}$ no longer depends on the symbol index, but only on the distance $D$.

If we plot the phase of the products $Y_{D, k}$ as a function of the pilot subcarrier index $k$, as long as the static channel hypothesis holds, we obtain a linear pattern with the previously neglect additive Gaussian noise as shown in Fig. 2.

The main goal is to determine the slope, which directly gives the corresponding $\zeta$ (SCO). In order to provide such estimate, let us consider the phase difference between two distinct arbitrary carriers $k_{1}$ and $k_{2}$, with $\Delta k=k_{1}-k_{2}$, so that

$$
\Delta B(D, \Delta k)=\arg \left(Y_{D, k_{1}} \cdot Y_{D, k_{2}}^{*}\right)=2 \pi \Delta k \zeta D N_{s} / N
$$

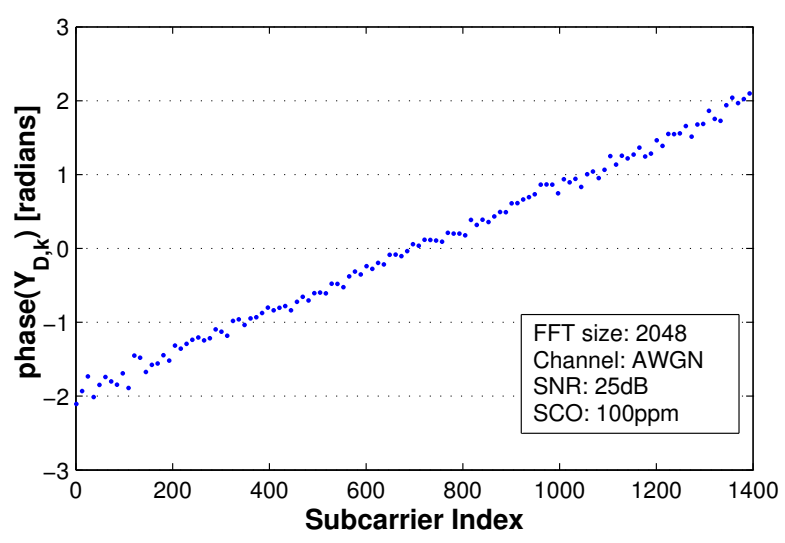

Fig. 2. Phase of the product $Y_{D, k}$ with a $25 \mathrm{~dB}$ SNR and an additive white Gaussian channel for 1404 active subcarriers and 604 virtual subcarriers, such as defined in the ISDB-T standard.

Now, considering the presence of noise that was neglected in eqs. (14) and (15), the best way to achieve a more robust estimation is to consider all possible combinations of two $Y_{D, k}$ and then calculate the average - always dividing by the corresponding carrier spacing $\Delta k$

$$
\hat{\zeta}=\frac{N}{2 \pi D N_{s}}\left(\frac{\sum_{\Delta k} \Delta B(D, \Delta k) / \Delta k}{M(M-1) / 2}\right)
$$

where $M$ is the total number of pilots in one OFDM symbol.

This is a one-shot estimator, and a new estimation can be obtained at every incoming symbol. Also, in order to avoid distortions on the estimated SCO value, the SCO deviation must be so that the largest pilot carrier spacing $\left(\Delta k_{\max }\right)$ must result in $\Delta B\left(D, \Delta k_{\max }\right)<2 \pi$. In other words,

$$
\zeta<\frac{N}{N_{S}} \frac{1}{\Delta k_{\max } D} .
$$

As mentioned before, one of the assumptions used in this method is that the channel is time-invariant within the interval of $D$ OFDM symbols. If such assumption is not met, the channel time variation will cause an extra phase rotation that can be different for each subcarrier and, in principle, cannot be distinguished from the SCO effect. As shown in Fig. 3 the referred linear pattern in the phase of $Y_{D, k}$ might get severely distorted.

To illustrate this point, Fig. 4 shows the output of the aforementioned method for a constant SCO value of $\zeta=50$ ppm and under a mobile channel TU6 (see table II) with a normalized Doppler frequency of $f_{d} T_{s}=0.0094$ which, for a carrier frequency $f_{c}=806 \mathrm{MHz}$, corresponds to 50 $\mathrm{km} / \mathrm{h}$ for a FFT size of 2048 on ISDB-T standard and infinite signal-to-noise ratio (SNR). Since no noise was added to the signal, it can be seen that the channel variation alone induces severe deviations in the output of the estimator.

An effective strategy is to post-process the estimator output by averaging the estimated values using a filter such as an exponential moving average filter (i.e., a loop-back filter) given by [4]

$$
\hat{\zeta}_{l+1}^{\prime}=\lambda \hat{\zeta}_{l}^{\prime}+(1-\lambda) \hat{\zeta}_{l}
$$


where $\lambda \in[0,1)$ is the forgetting factor, in which the closest to one, the larger is the (exponentially decreasing) weight used to ponder the older estimations, $\hat{\zeta}_{l}$ and $\hat{\zeta}_{l}^{\prime}$ are the estimated SCO obtained from (16) and the exponential moving average filter output for the $l$-th OFDM symbol, respectively.

The averaged SCO guarantees a more precise result at the expense of some convergence delay. The exponential moving average filter output is also plotted in Fig. 4 and we can see that, although better behaved, its output is still quite noisy on a mobile environment, despite the large forgetting factor and the infinite SNR.

The resulting estimator structure is shown in Fig. 5.

\section{B. Mitigation of Time-Varying Channel Effects}

The phase distortion caused by the channel variation is different on each subcarrier. Some might be more distorted than others. For instance, in Fig. 3 the central subcarriers are quite far away from the line with the SCO slope. This observation is the central idea of the improvement we are proposing. Simply put, the method consists of a heuristic to identify the probable outliers - the most distorted terms - thus preventing them from contaminating the estimation.

In eq. (16) all possible combinations of $Y_{D, k}$ are considered in the averaging, even the ones that involve an outlier. Instead, we can set a certain threshold $\delta$ around a reference value and ignore all the $\Delta B(D, \Delta k) / \Delta k$ that would lead to a result outside this zone, as illustrated in Fig. 6. A similar idea has been proposed by Won et al. [14]. They performed an initial estimation by the conventional method (given by eq. (16)) and took it as a reference for identifying the outliers in the set of all possible $\Delta B(D, \Delta k) / \Delta k$. Then, the outliers are eliminated and the estimation is recalculated without them. However, some realizations of the set $\Delta B(D, \Delta k) / \Delta k$ may be severely distorted and the classical estimation will be very far from the actual SCO. So, taking it as a reference would result in a poor identification of the outliers. Then our first contribution, firstly shown in [1], is to avoid this problem by using the exponential moving average output, $\hat{\zeta}^{\prime}$, as the reference since it provides a more reliable estimation

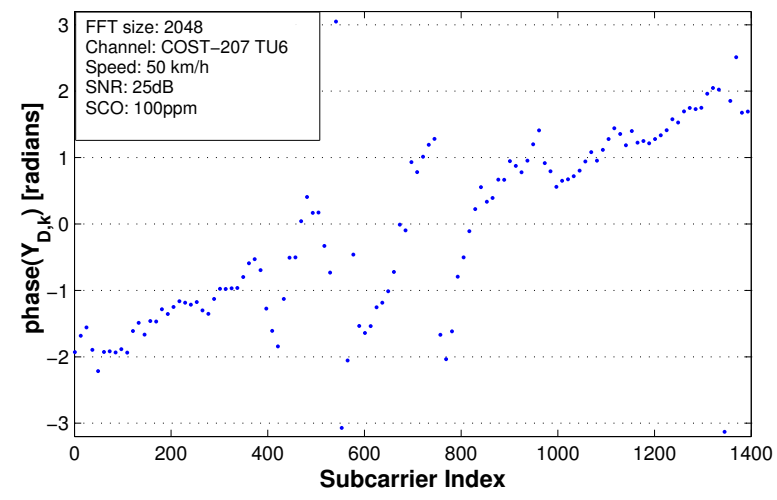

Fig. 3. Phase of the product $Y_{D, k}$ on TU6 channel $50 \mathrm{~km} / \mathrm{h}$ and $25 \mathrm{~dB}$ SNR for 1404 active subcarriers and 604 virtual subcarriers, such as defined in the ISDB-T standard.

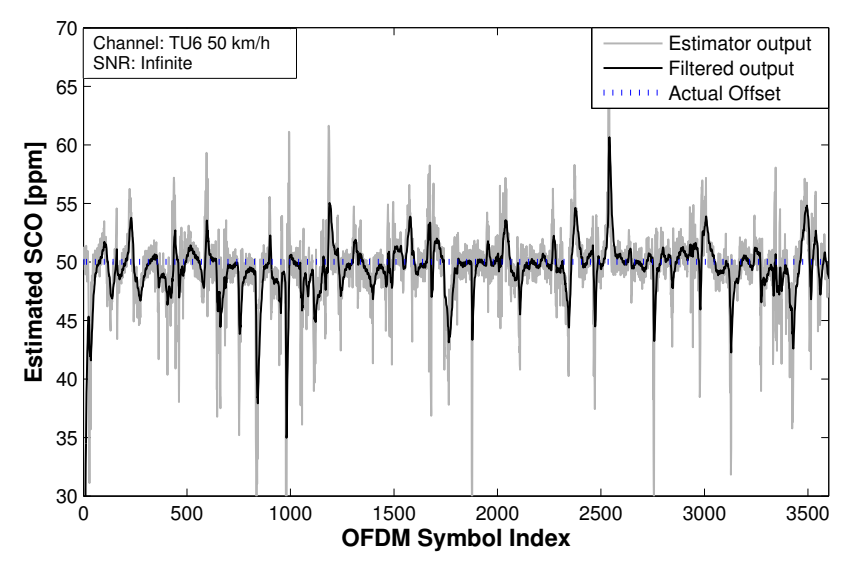

Fig. 4. Conventional output of the estimator (eq. (16)) on a TU6 channel $50 \mathrm{~km} / \mathrm{h}$ (ISDB-T, $N=2048$ ) and infinite SNR. The forgetting factor of the filtered output is equal to 0.9 .

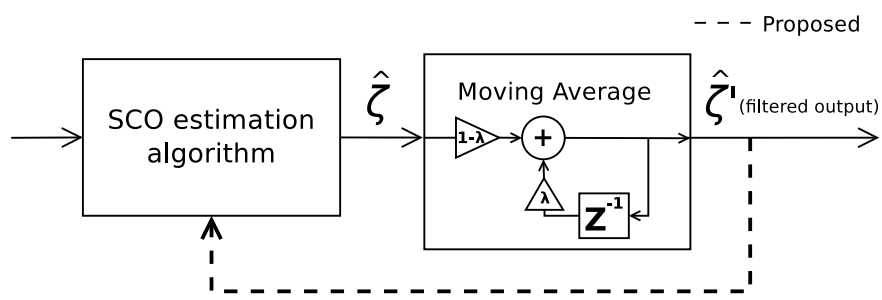

Fig. 5. Estimation block diagram. The dashed line is added by the proposed method.

by averaging over many other realizations. This modification corresponds to the dashed line in Fig. 5. A case where the two mentioned reference values differ significantly is shown in Fig. 6. Since the illustrated realization is severely distorted, the approach proposed in [14] gets biased by the numerous outliers while the proposed approach leads to a much more accurate reference value.

Notwithstanding the good steady-state results of this approach, as shown in [1], it also presents a problem. In the event of an abrupt change of the SCO with respect to the reference value, such as in the system initialization where the

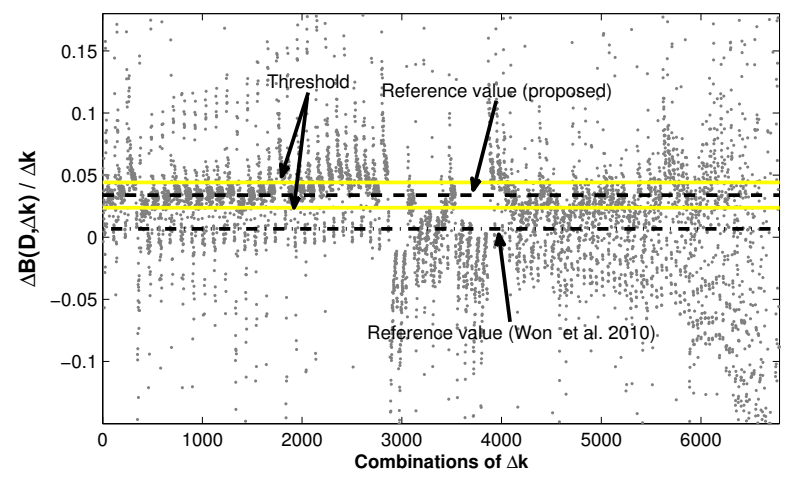

Fig. 6. Threshold application on the values of $\Delta B(D, \Delta k)$ on TU6 channel $50 \mathrm{~km} / \mathrm{h}$ and $25 \mathrm{~dB}$ SNR. 
reference value is initialized with zero, it is possible that the entire set of $\Delta B(D, \Delta k) / \Delta k$ gets discarded, generating a null $\hat{\zeta}$. In order to overcome such an exception, we make $\hat{\zeta}=\hat{\zeta}^{\prime}$, i.e., we feed the moving average filter with its own output and thus, we keep the same $\hat{\zeta}^{\prime}$ as a reference, since we do not have a valid estimation. If such scenario is repeated indefinitely, the estimator proposed in [1] will not converge to the true SCO value.

In order to overcome such limitation, additional mechanisms can be introduced. Switching from the conventional to the proposed method in [1] after quite a few OFDM symbols solves the problem for the system initialization, but not for an abrupt change of the SCO after it, as we illustrate further ahead. A possibility is to use an adaptive threshold, but the calculation of such value is not evident and can become very cumbersome. We avoid such problem by providing two simple methods that can achieve similar or better performances with respect to our original solution in [1] and that is also robust to any abrupt deviation of the actual SCO.

The first method is to saturate to the closest threshold every value $\Delta B(D, \Delta k) / \Delta k$ that violates it. The saturation algorithm is described in Algorithm 1

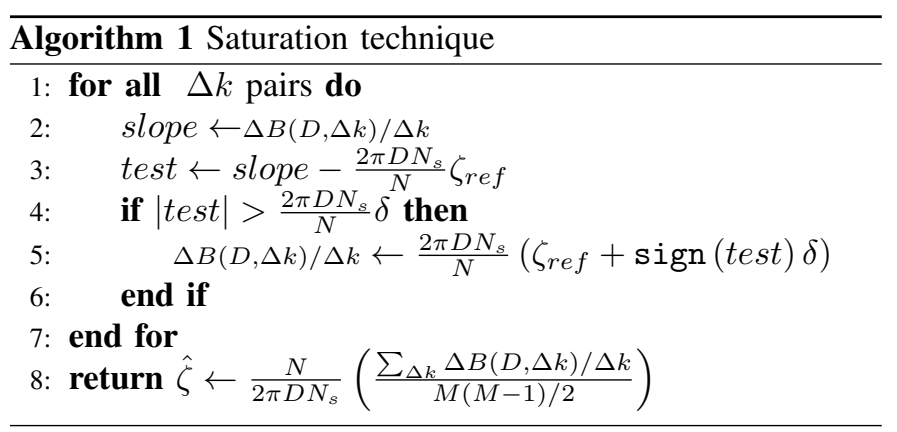

The other technique is to use a constrained set formed by the $Q$ values among all possible $\Delta B(D, \Delta k) / \Delta k$ values that are the closest to the reference $\frac{2 \pi D N_{s}}{N} \zeta_{\text {ref }}$. Such technique is described in Algorithm 2

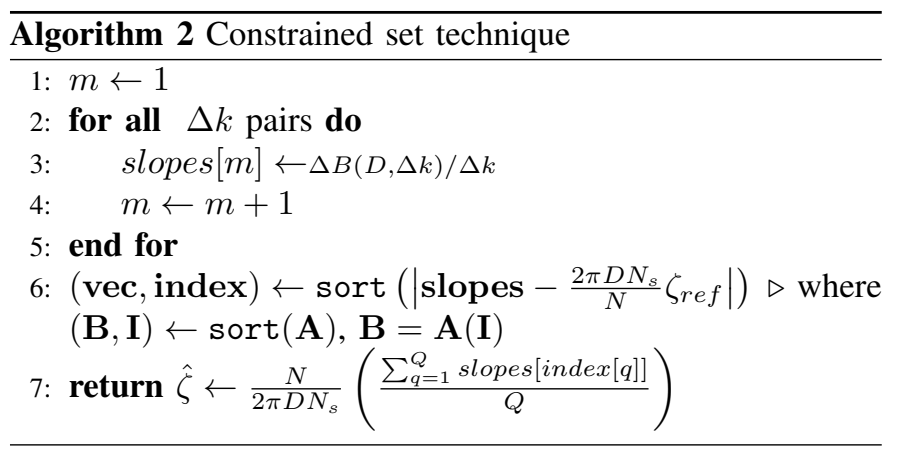

Section IV presents some simulation results confirming the superiority of our proposed method. It is worth noting that, besides the technique briefly described here in Section III-B and in more detail in [14], Won et al. also proposes other measures to achieve better performance such as a noise reduction technique through the use of the channel impulse response sparsity. These other improvements are left aside when implementing the Won technique in order to make a fair
TABLE I

SIMULATION PARAMETERS

\begin{tabular}{|l|l|}
\hline FFT size & 2048 \\
\hline Guard interval & $1 / 8$ \\
\hline Pilot symbols per OFDM symbol & 117 \\
\hline Pilot symbols spacing & 12 \\
\hline Number of virtual subcarriers & 604 \\
\hline Comb pilot pattern period $(D)$ & 4 \\
\hline System bandwidth & $6 \mathrm{MHz}$ \\
\hline Carrier Frequency & $806 \mathrm{MHz}$ \\
\hline Data Modulation & $64 \mathrm{QAM}$ \\
\hline Channel Profile & TU6 (see Table II) \\
\hline Threshold $(\delta)$ & $6 \mathrm{ppm}$ \\
\hline Constrained set size $(Q)$ & 1000 \\
\hline
\end{tabular}

comparison regarding only the additional noise introduced by the time-varying channel.

\section{Simulation Results}

The simulations parameters based on the ISDB-T standard are listed in Table I (except for the System validation part). The threshold value $(\delta)$ and the constrained set size $(Q)$ were obtained through simulations for a forgetting factor of 0.9 aiming a good performance compromise for both mild and high mobility scenarios $(50 \mathrm{~km} / \mathrm{h}$ and $200 \mathrm{~km} / \mathrm{h})$ in terms of root mean square error (RMSE), i.e.

$$
\operatorname{RMSE}=\sqrt{\frac{1}{N} \sum_{k=0}^{N-1}\left(\zeta-\hat{\zeta}_{k}^{\prime}\right)^{2}}
$$

where $N$ is the number of estimations and $\hat{\zeta}_{k}^{\prime}$ is the SCO estimation after the moving average filtering for the $k$-th OFDM symbol. For more details on the choice of the threshold value and the constrained set size, refer to subsection IV-A.

The mobile channel profile is the TU6 (6-tap Typical Urban) defined by COST-207 standard [15] and described in Table II. Finally, the pilot symbol power is $4 / 3$ of the average data symbol power as defined by [3].

TABLE II

TU6 CHANNEL PARAMETERS

\begin{tabular}{|c|c|c|}
\hline \#Tap & Delay [us] & Power [dB] \\
\hline 1 & 0.0 & -3 \\
\hline 2 & 0.2 & 0 \\
\hline 3 & 0.5 & -2 \\
\hline 4 & 1.6 & -6 \\
\hline 5 & 2.3 & -8 \\
\hline 6 & 5.0 & -10 \\
\hline
\end{tabular}

System Validation: In order to validate our simulations results, we have reproduced some previously published results of a SCO estimator's performance under the same simulation setup. In [16] the conventional method has been implemented on a ISDB-T system with the slight modification of fixing a single carrier spacing $\Delta k$ instead of averaging over all possible spacings $\Delta k$ as in eq. (16). By using the same simulation parameters as in [16] (Table II), we obtain very close RMSE results as shown in Fig. 7. 


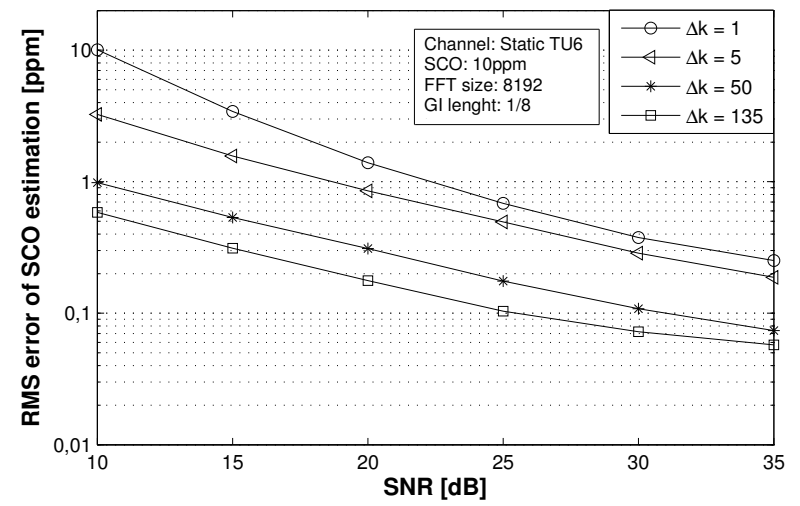

Fig. 7. RMSE vs. SNR for the conventional method with fixed $\Delta k$ under the same simulation scenario as in [16].

Performance analysis: Firstly, using a forgetting factor equal to 0.9 for all techniques, i.e., the conventional one, the method proposed by Won et al. [14], the saturation method and the constrained set, as well as the version proposed by us in [1], we evaluate their RMSE x SNR performance for $6 \mathrm{~km} / \mathrm{h}$ (Doppler spread $f_{d}=4.48 \mathrm{~Hz}$ and normalized Doppler spread $f_{d} T_{s}=0.0011$ for the specified parameters $), 50 \mathrm{~km} / \mathrm{h}\left(f_{d}=\right.$ $37.31 \mathrm{~Hz}$ and $\left.f_{d} T_{s}=0.0094\right)$ and $200 \mathrm{~km} / \mathrm{h}\left(f_{d}=149.26\right.$ $\mathrm{Hz}$ and $\left.f_{d} T_{s}=0.0376\right)$ in Figs. 8, 9 and 10, respectively. The RMSE values where obtained by using ten independent runs with a total of 3600 OFDM symbols each, in which 250 symbols were used to eliminate any transient behavior of the exponential moving average filter.

We can see that all techniques suffer from an error floor for higher SNR values, but the proposed techniques are much more effective for higher mobility channels (i.e., $200 \mathrm{~km} / \mathrm{h}$ ), providing more than three times less RMSE than the Won. et al. [14] and the conventional technique, which have similar performances. For mild mobility channels (i.e., $50 \mathrm{~km} / \mathrm{h}$ ), the performance difference is smaller as would be expected, since the distortion introduced by the time-varying channel is naturally mitigated. Nonetheless, the constrained set technique presents some advantage over the saturation one for the lower

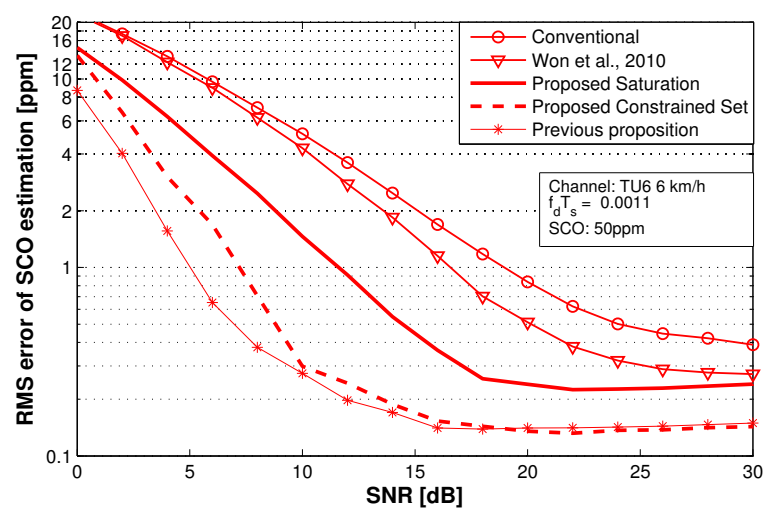

Fig. 8. RMSE vs. SNR (TU6 channel $6 \mathrm{~km} / \mathrm{h}$ )

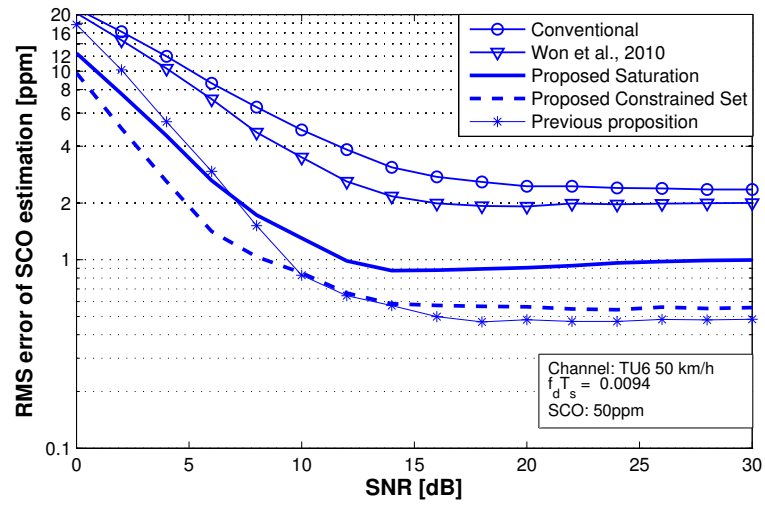

Fig. 9. RMSE vs. SNR (TU6 channel $50 \mathrm{~km} / \mathrm{h}$ )

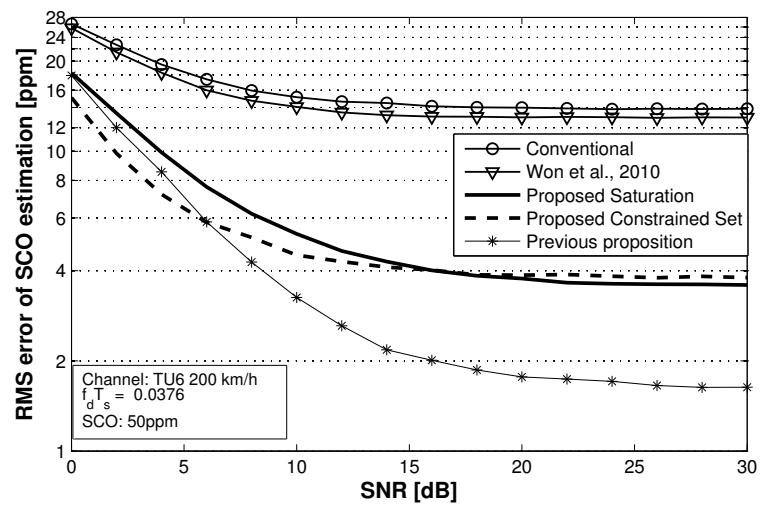

Fig. 10. RMSE vs. SNR (TU6 channel 200 km/h)

range of SNR values.

The two techniques proposed in this article achieved considerably close results when compared to the simplified version previously proposed, except on the high speed scenario. In any case, it is important to emphasize that the previous technique is not sufficiently robust as will be demonstrated in the following.

One may correctly argue that the higher RMSE of the conventional and Won et al estimators with regard to the proposed techniques can be equalized with additional filtering such as using a larger forgetting factor. However, this will negatively affect the convergence rate, making it slower. Hence, in order to analyze such question, we have adjusted the forgetting factor of all techniques in order to make their steady-state RMSE equal. The RMSE obtained by the saturation technique with a forgetting factor of 0.9 was taken as a reference. The forgetting factors required by each technique to equalize such reference is shown in Table III.

Then, we present the estimators' outputs along the time (in OFDM symbols) for a fixed SNR of $20 \mathrm{~dB}$ and an abrupt change of $100 \mathrm{ppm}$ of the SCO in Figs. 11, 12 and 13 for 6, 50 and $200 \mathrm{~km} / \mathrm{h}$, respectively.

Table IV shows the number of symbols required by each technique to converge, averaged over ten independent channel realizations. The estimator is considered to have converged as 


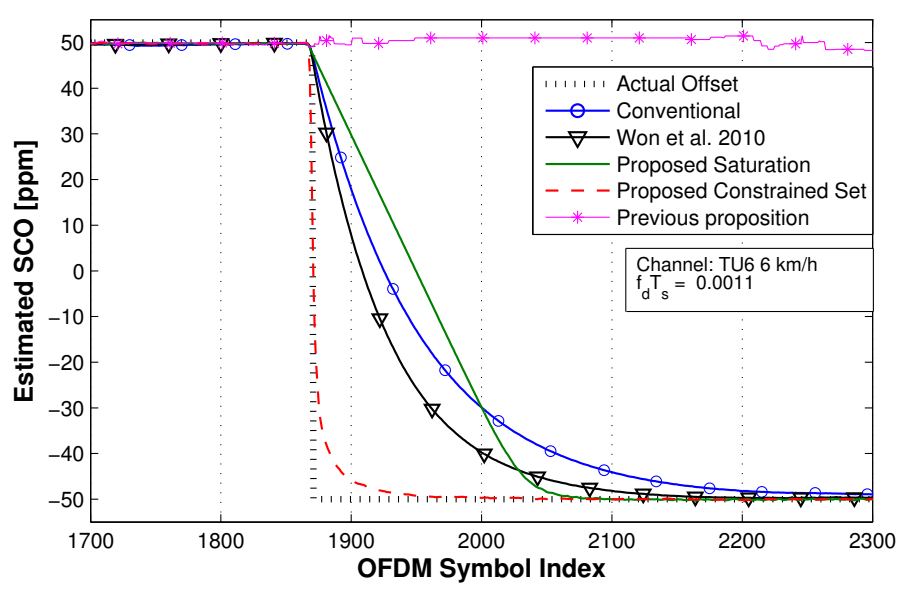

Fig. 11. Convergence rate comparison (TU6 channel $6 \mathrm{~km} / \mathrm{h}$ )

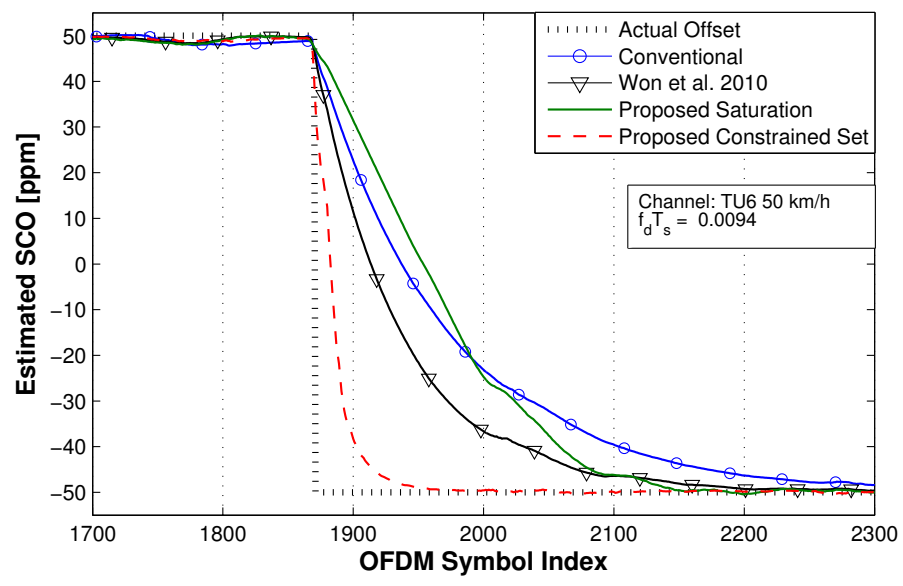

Fig. 12. Convergence rate comparison (TU6 channel $50 \mathrm{~km} / \mathrm{h}$ )

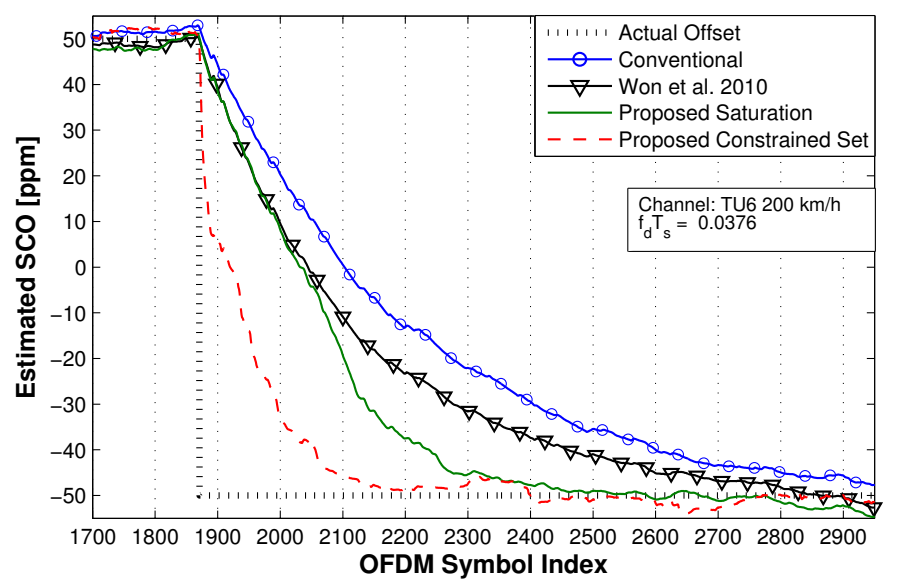

Fig. 13. Convergence rate comparison (TU6 channel $200 \mathrm{~km} / \mathrm{h}$ ) soon as its output reaches an interval of $5 \mathrm{ppm}$ around the actual offset.

It can be seen in Fig. 11 that the simplified technique proposed in [1] could not cope with an abrupt change in the SCO, as anticipated in Section III-B. For this reason, this particular technique will be omitted from the remaining comparisons.

For the speeds of $6 \mathrm{~km} / \mathrm{h}$ and $50 \mathrm{~km} / \mathrm{h}$, the convergence rate simulation results show that the conventional is the slowest and the Won et al. and saturation methods present about the same convergence rate and only a small improvement over the conventional estimator. However, the constrained set is able to provide a considerable advantage over the other techniques. For the high mobility scenario of $200 \mathrm{~km} / \mathrm{h}$, the saturation and constrained set techniques are substantially faster than the other two. The constrained set is still the fastest one, but its advantage is less pronounced than in the other speed scenarios. Note also that the convergence rate of the saturation method is for the first few iterations the slowest one, since its SCO estimative is limited to $\pm \delta \mathrm{ppm}$ of the reference value $\hat{\zeta}_{l}^{\prime}$. It is worth noting that this is less of a problem for lower SCO abrupt deviations, like 50 ppm instead of 100 ppm and mild mobility scenarios, where the saturated method convergence rate is considerably improved with regard to the conventional and Won et al. methods as illustrated in Fig. 14. The explanation comes from the fact that the bounded estimative is initially closer to the true one, resulting in a smaller estimation error, while, despite being able to initially estimate the true SCO deviation (with additional noise), the conventional and the Won et al. methods still require a larger number of iterations due to the larger forgetting factors needed to achieve the same steady-state RMSE of the saturated method. And again, the constrained set still offers the fastest convergence.

Finally, it must be mentioned that the advantage of the constrained set over the other methods comes at the expense of some additional complexity due to selecting, among all $N_{p}\left(N_{p}-1\right) / 2$ slopes, the $Q$ closest to the reference value. As formulated in Algorithm 2, it corresponds to an unordered partial sorting problem, which consists of returning the $Q$ smallest elements of a vector, but not necessarily in order. The computational cost associated to such a task, using the quickselect algorithm, is linear on the size of the set to be

TABLE III

ForGETTING FACTORS USED TO ACHIEVE THE SAME RMSE PERFORMANCE

\begin{tabular}{|l|c|c|c|c|}
\cline { 2 - 5 } \multicolumn{1}{c|}{} & Convetional & Won et al. & Saturation & Constrained set \\
\hline $6 \mathrm{~km} / \mathrm{h}$ & 0.988 & 0.983 & 0.9 & 0.6 \\
\hline $50 \mathrm{~km} / \mathrm{h}$ & 0.99 & 0.985 & 0.9 & 0.6 \\
\hline $200 \mathrm{~km} / \mathrm{h}$ & 0.996 & 0.995 & 0.9 & 0.91 \\
\hline
\end{tabular}

TABLE IV

AVERAGE CONVERGENCE TIME IN OFDM SYMBOLS

\begin{tabular}{|l|c|c|c|c|}
\cline { 2 - 5 } \multicolumn{1}{c|}{} & Conventional & Won et al. & Saturation & Constrained set \\
\hline $6 \mathrm{~km} / \mathrm{h}$ & 250 & 171 & 171 & $\mathbf{2 6}$ \\
\hline $50 \mathrm{~km} / \mathrm{h}$ & 311 & 206 & 202 & $\mathbf{4 5}$ \\
\hline $200 \mathrm{~km} / \mathrm{h}$ & 959 & 768 & 411 & $\mathbf{2 0 9}$ \\
\hline
\end{tabular}




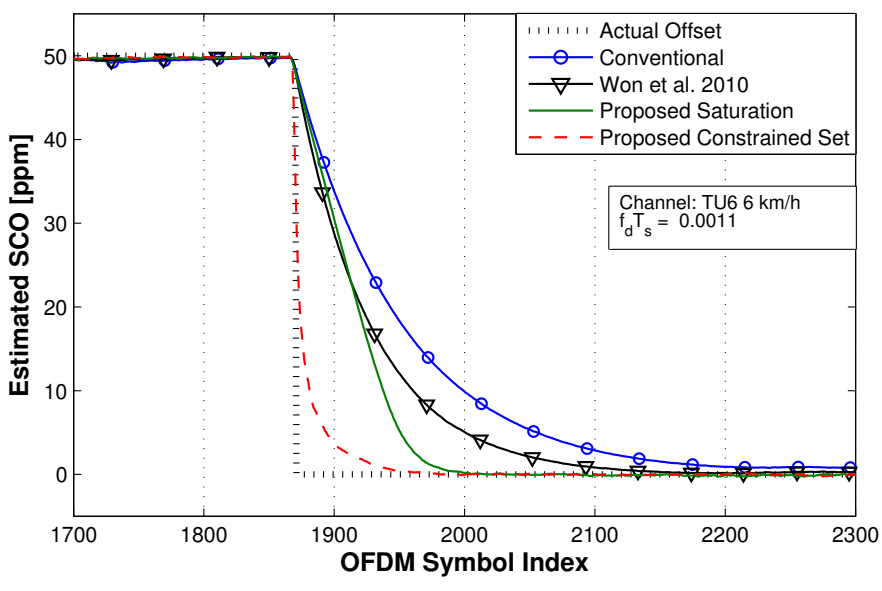

Fig. 14. Convergence rate comparison (TU6 channel $6 \mathrm{~km} / \mathrm{h}$ ) with a $50 \mathrm{ppm}$ SCO change.

partially ordered [17] i.e. about $\mathcal{O}\left(\frac{N_{p}^{2}}{2}\right)$.

It is also worth to use the BER as a performance metric. In order to avoid ICI, which arises from the channel varying during the OFDM symbol, and the need to add cancelation techniques like [18], [19] to avoid a high error floor, we adopted a block-fading scheme. In such a case, the channel coefficients are kept constant over an OFDM symbol, but varies accordingly to the Jakes model from one symbol to another one, so that the perturbation on the SCO estimations is still present. Also, in order to make the results more practical, we used a convolutional code in octal representation [133 171] as a forward error correction code. The estimated channel that is used to demodulate the data is obtained independently in each OFDM symbol (one-shot basis) using the least square criterion on the pilot symbols followed by linear interpolation. The forgetting factor used to average the SCO estimation was 0.6 for all techniques. A Farrow interpolator is used to compensate the SCO deviation obtained through the estimator. The results are shown in Fig. 15.

As we can see from the BER results depicted in Fig. 15, both proposed techniques presents the same performance for both scenarios. With regard to the Won et al. technique, for a target BER of $10^{-4}$, the proposed techniques outperform it by $0.5 \mathrm{~dB}$ for $200 \mathrm{~km} / \mathrm{h}\left(f_{d} T=0.0376\right)$ and by $2 \mathrm{~dB}$ for $300 \mathrm{~km} / \mathrm{h}$ $\left(f_{d} T=0.0564\right)$. Also, the advantage of Won et al. with respect to the conventional one is very small in both scenarios and both of them hit an error floor while the proposed techniques can achieve a much lower BER before also hitting an error floor. In addition, the lower the forgetting factor (used for faster acquisition and tracking of the SCO deviation) the higher is the sensitivity to the normalized Doppler frequency.

It is worth noting that the simulated speeds might seem unrealistically high, but their corresponding normalized Doppler frequencies can be easily attained in other configurations of the very same television standard. In fact, in other transmission modes the symbol size may be increased by a factor of two or even four while keeping the sampling frequency unchanged. This implies attaining the

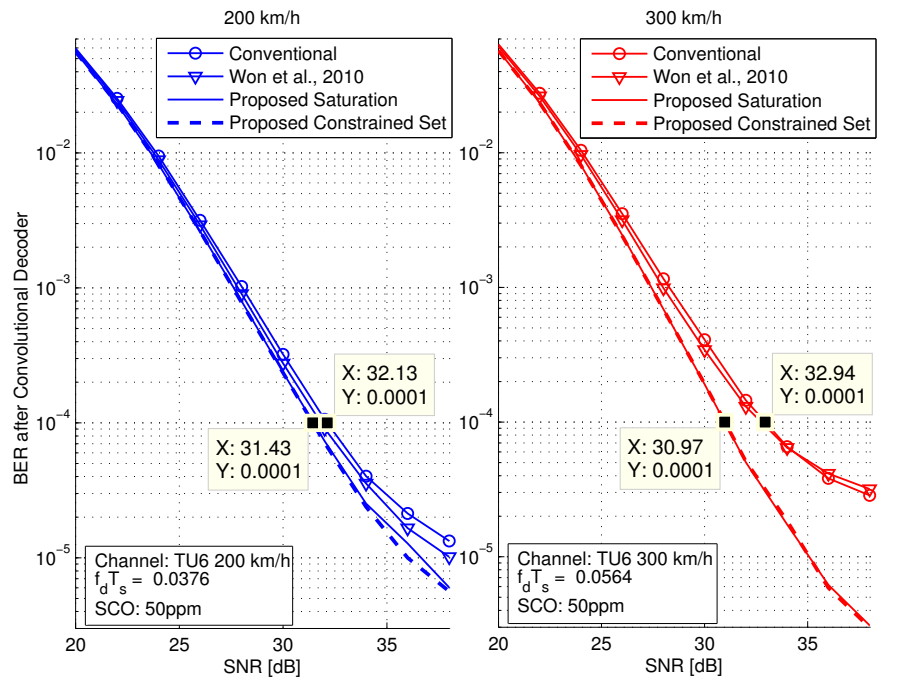

Fig. 15. BER versus SNR comparison for TU6 channel and $200 \mathrm{~km} / \mathrm{h}\left(f_{d} T=\right.$ $0.0376)$ and $300 \mathrm{~km} / \mathrm{h}\left(f_{d} T=0.0564\right)$ scenarios with a $50 \mathrm{ppm}$ SCO.

same normalized Doppler frequencies at speeds two or four times smaller.

\section{A. Choosing the Threshold Value and the Constrained Set Size}

Similar to the forgetting factor, these two parameters are a form of controlling the trade-off between the estimator's accuracy and its convergence speed. Depending on the system requirements, one of the features may be prioritized. In order to formalize this compromise, we have measured, for a wide range of each parameter, both the RMSE on a constant offset and the convergence rate for an offset change of $100 \mathrm{ppm}$. The forgetting factor was fixed at 0.9 and the SNR at $20 \mathrm{~dB}$ for this analysis.

The threshold $(\delta)$ was varied from $1 \mathrm{ppm}$ to $100 \mathrm{ppm}$, and the constrained set size $(Q)$ from 400 to 4000 . For values below such ranges, the estimators convergence rate gets prohibitively slow. Likewise, for bigger values almost no RMSE improvement with respect to the conventional method is observed. On a RMSE vs. Convergence speed graph, a particular trade-off curve is defined by sweeping each of these two parameters, as shown in Fig. 16. Some specific parameter values are identified on the figure as a reference.

Based on the graphs, the system designer can freely choose the parameter values that provides him the desired trade-off. But typically, for this kind of problem, a reasonable solution would lie close to the lower-left corner of the trade-off curve. Our choice of parameters for the previous simulations was based on this principle. Nevertheless, a particular choice might not be optimal for all speed scenarios. It is also worth noting that values of $Q$ smaller than 800 are not an interesting choice, since in this region of the curve the RMSE slightly grows.

\section{CONCLUSION}

In this paper we proposed two methods to improve the conventional pilot-based SCO estimation algorithm in the context of a mobile environment. In this scenario some pilots 


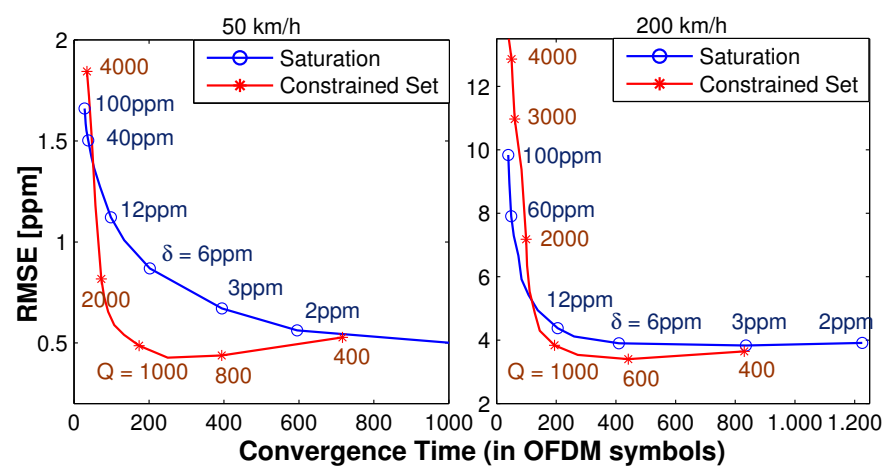

Fig. 16. Trade-off curves between RMSE and Convergence rate on TU6 channel $50 \mathrm{~km} / \mathrm{h}$ (left) and $200 \mathrm{~km} / \mathrm{h}$ (right).

may suffer severe phase distortion due to the time-varying channel, which considerably degrades the SCO estimation. Our proposal consists of two heuristic methods that identify the least reliable data and substitute them by bounded estimates or just eliminate them from the calculation of the SCO in order to improve it. Simulation results have shown a considerable RMSE reduction for all ranges of SNR, specially for higher Doppler spread or faster convergence rates in comparison to the conventional method and another similar technique with none or little additional computational complexity.

\section{ACKNOWLEDGEMENTS}

The authors would like to thank the National Council for Scientific and Technological Development (CNPq) for the support (454410/2013-1), as well as Idea! Electronic Systems.

\section{REFERENCES}

[1] C. Dantas, D. Castro, and C. Panazio, "Improvement on sampling clock offset estimation for mobile OFDM systems," in XXXIII Brazilian Telecommunications Symposium (SBrT), 2015, pp. 557-561.

[2] ETSI, EN 300 744, v1.5.1 ed., Digital Video Broadcasting (DVB); Framing structure, channel coding and modulation for digital terrestrial television, Jun. 2004.

[3] ARIB, ARIB STD-B31 Standard, v1.6-E2 ed., Transmission System for Digital Terrestrial Television Broadcasting Association of Radio Industries and Businesses, Nov. 2005.

[4] M. Speth, S. Fechtel, G. Fock, and H. Meyr, "Optimum receiver design for OFDM-based broadband transmission .II. A case study," IEEE Transactions on Communications, vol. 49, no. 4, pp. 571-578, 2001. doi: http://dx.doi.org/10.1109/26.917759

[5] W. Dan and H. A. qun, "A combined residual frequency and sampling clock offset estimation for OFDM systems," in IEEE Asia Pacific Conference on Circuits and Systems (APCCAS), 2006, pp. 1184-1187. doi: http://dx.doi.org/10.1109/APCCAS.2006.342353

[6] M. Sliskovic, "Carrier and sampling frequency offset estimation and correction in multicarrier systems," in IEEE Global Telecommunications Conference (GLOBECOM), vol. 1, 2001, pp. 285-289 vol.1. doi: http://dx.doi.org/10.1109/GLOCOM.2001.965124

[7] Y.-H. You, J. Kim, and H.-K. Song, "Pilot-assisted fine frequency synchronization for OFDM-based dvb receivers," IEEE Transactions on Broadcasting, vol. 55, no. 3, pp. 674-678, Sept 2009. doi: http://dx.doi.org/10.1109/TBC.2009.2027400

[8] Y. Wu, Y. Zhao, and D. Li, "Sampling frequency offset estimation for pilot-aided OFDM systems in mobile environment," Wireless Personal Communications, vol. 62, no. 1, pp. 215-226, 2012. doi: http://dx.doi.org/10.1007/s11277-010-0049-x
9] E. Oswald, "NDA based feedforward sampling frequency synchronization for OFDM systems," in IEEE 59th Vehicular Technology Conference, VTC-Spring, vol. 2, May 2004, pp. 1068-1072 Vol.2. doi: http://dx.doi.org/10.1109/VETECS.2004.1388995

[10] A. Laourine, A. Stephenne, and S. Affes, "Blind sampling clock offset estimation in OFDM systems based on second order statistics," in Fortieth Asilomar Conference on Signals, Systems and Computers (ACSSC), Oct 2006, pp. 1782-1785. doi: http://dx.doi.org/10.1109/ACSSC.2006.355068

[11] M. Speth, S. A. Fechtel, G. Fock, and H. Meyr, "Optimum receiver design for wireless broad-band systems using OFDM. I," IEEE Transactions on Communications, vol. 47, no. 11, pp. 1668-1677, 1999. doi: http://dx.doi.org/10.1109/26.803501

[12] W. C. Jakes, Microwave Mobile Communications. New York: Wiley, 1974.

[13] Y. H. Kim, I. Song, H. G. Kim, T. Chang, and H. M. Kim, "Performance analysis of a coded OFDM system in time-varying multipath Rayleigh fading channels," IEEE Transactions on Vehicular Technology, vol. 48, no. 5, pp. 1610-1615, Sep 1999. doi: http://dx.doi.org/10.1109/25.790538

[14] K. H. Won, J. Han, and H. Choi, "Sampling frequency offset estimation methods for DVB-T/H systems," Journal of Networks, vol. 5, no. 3, pp. 313-320, 2010. doi: http://dx.doi.org/10.4304/jnw.5.3.313-320

[15] COST 207 Digital Land Mobile Radio Communications: Final Report, 14 March 1984, 13 September 1988, ser. Euratom publications - EUR. Office for Office Publ. of the European Communities, 1989.

[16] N. Surantha, Y. Nagao, M. Kurosaki, and H. Ochi, "A computationally efficient sampling frequency offset estimation for ofdm-based digital terrestrial television systems," in 2013 IEEE 24th Annual International Symposium on Personal, Indoor, and Mobile Radio Communications (PIMRC), Sept 2013, pp. 662-666. doi: http://dx.doi.org/10.1109/PIMRC.2013.6666219

[17] B. Vallee, J. Clement, J. Fill, and P. Flajolet, "The number of symbol comparisons in quicksort and quickselect," in Automata, Languages and Programming, ser. Lecture Notes in Computer Science, S. Albers, A. Marchetti-Spaccamela, Y. Matias, S. Nikoletseas, and W. Thomas, Eds. Springer Berlin Heidelberg, 2009, vol. 5555, pp. 750-763. doi: http://dx.doi.org/10.1007/978-3-642-02927-1_62

[18] P. Schniter, "Low-complexity equalization of OFDM in doubly selective channels," IEEE Transactions on Signal Processing, vol. 52, no. 4, pp. 1002-1011, April 2004. doi: http://dx.doi.org/10.1109/TSP.2004.823503

[19] Y. Mostofi and D. C. Cox, "ICI mitigation for pilot-aided OFDM mobile systems," IEEE Transactions on Wireless Communications, vol. 4, no. 2, pp. 765-774, March 2005. doi: http://dx.doi.org/10.1109/TWC.2004.840235

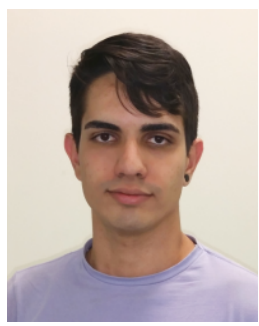

Cássio F. Dantas obtained a double degree in Electrical Engineering at University of Campinas (Bachelor) and École Polytechnique de Paris (Master) in 2014. He has two years of R\&D experience on Telecommunications and Digital Signal Processing at Idea! Electronic Systems. Currently, he is a MSc student at DSPCom laboratory, University of Campinas. His research interests lie on the frontier between signal processing and machine learning.

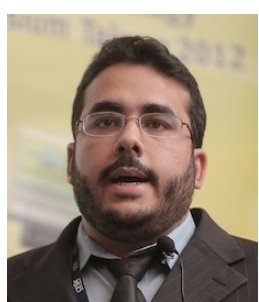

Davi A. L. Castro was born in Maceió, Alagoas, Brazil in 1984. He obtained the degree of Electrical Engineer in 2009 from the Federal University of Campina Grande (UFCG), Brazil. He specialized in integrated circuit design through CI-Brasil training program in 2009. Since 2010 he has been working in $\mathrm{R} \& \mathrm{D}$ for telecommunication and digital TV semiconductor products at Idea! Electronic Systems. 
Cristiano Panazio received a B.Sc. and an M.Sc. degree in electrical engineering from the State University of Campinas (UNICAMP), Brazil, in 1999 and 2001, respectively. He received his Ph.D. in 2005, also in electrical engineering, from the Conservatoire National des Arts Métiers (CNAM), Paris, France. In 2006, he became Assistant Professor at Escola Politécnica of the University of São Paulo (EPUSP). He is member of the Brazilian Telecommunications Society (SBrT) since 1999. His research interests include equalization, multicarrier modulations, synchronization techniques and cognitive radio. 